\title{
Determination the capacity of two-lane suburban roads with neural networks and effect of speed on level of service
}

\author{
Mahmoud Ameri • Reza Moayedfar • Fatemeh Jafari
}

Received: 16 September 2012 / Accepted: 6 February 2013 /Published online: 17 March 2013

(C) The Author(s) 2013. This article is published with open access at SpringerLink.com

\begin{abstract}
Introduction This study aimed to determine the capacity of two-lane suburban roads and its effect on level of service.

Materials and methods Traffic data used in this study prepared from statistics available at the site of road transport Organization and has been tried that the roads are selected from the various regions of level terrain, rolling terrain and mountainous. Because of advantage of the neural network approach than the classic methods, it is selected for determining of capacity.

Results As a result, the 5-7-1 structure was selected as the best structure to determine capacity. The method which is presented in HCM 2000 manual was used to determine the level of service. This method determines the level of service based on percent time spent following (PTSF) and average travel speed (ATS).

Conclusions The results show that level of service based on average travel speed is better than the level of service based on percent time spent following and this is indicative that drivers move with higher average speed than prevailing condition and it cause to decrease safety.
\end{abstract}

\footnotetext{
M. Ameri

Civil Engineering, Transportation Research Institute, Center of Excellence for PMS, Transportation and Safety, Iran University of Science and Technology, Tehran, Iran

R. Moayedfar $(\bowtie)$

Transportation Engineering, Faculty of Engineering, Arak

University, Arak, Iran

e-mail: r_moayedfar@yahoo.com

R. Moayedfar

e-mail: r-moayedfar@araku.ac.ir

F. Jafari

Transportation Engineering, Faculty of Engineering, Islamic Azad University, South of Tehran Branch, Tehran, Iran
}

Keywords Capacity $\cdot$ Level of service $\cdot$ The neural network · Percent time spent following

\section{Introduction}

Today, one of the most critical needs of traffic engineering is a clear understanding of the capabilities of specified facility. For about how much traffic and under what functional conditions can be coordinated. If engineers estimate the ability of facilities is less than its actual capacity, it requires to design over excessive need and causes to waste capital and resources. For better understanding the importance and necessity of control knowledge and efficient use of network communication, It is enough to point out the types of respiratory and audio infections, large-scale loss of time for users of the network, as well as problems and neurological disorders for drivers and passengers and the incidence of accidents and unforeseen events, and generally disturbing the public order and citizen's lives [1].

HCM 2000 defines the capacity as:

Capacity of a route is the maximum hourly rate at which persons or vehicles can move in a reasonable order of a point or a lane of road, during a period of time under the prevailing conditions of path, traffic and control [2].

Parameters affecting capacity include traffic conditions, the properties related to geometric design-the control conditionspavement conditions [3], weather conditions and light [4], local conditions, characteristics and culture of driver [5].

In determining the level of service, quality terms of traffic flow at the point of drivers and passengers should be measured $[6,7]$.

\section{History of research}

For determination of capacity based on HCM2000 manual, first of all has been estimated capacity of two-lane roads in 
ideal condition in two directions of $3,200 \mathrm{pc} / \mathrm{h}$ and in one direction $1,700 \mathrm{pc} / \mathrm{h}$ and has been determined the ideal conditions below: 1 . Width of each lane should be $12 \mathrm{ft}$ or more. 2. Width of shoulders should be $6 \mathrm{ft}$ or more. 3. The road should be in level terrain. 4. there is no heavy vehicle. 5. No passing zone exist.6. Traffic directional distribution is 50/50. 7. Traffic is not interrupted. After estimating the capacity of two-lane roads by using decreasing indexes, which caused by traffic, geometrical and control conditions, the capacity is calculated. Tables and diagrams have been provided by simulation software. Level of service is categorized in A to F, which $\mathrm{A}$ indicates the best conditions and $\mathrm{F}$ indicates the worst condition of traffic. For determining the service level we have used two parameters of percent time spent following (PTSF) and average travel speed (ATS) [8].

In the latest research which was done in Germany, after collecting necessary statistical data, speed-traffic volume diagram has been drawn by simulation software in different topography conditions, from direct and unisonous roads in smooth regions, to tortuous roads in mountainous regions, and finally the relevant amounts of capacity has been took. With regard to the results, capacity of two-lane roads in smooth and unisonous conditions in Germany had been determined 2,500 vehicles in an hour, in which this amount in slope roads and the roads with horizontal arch has been decreased to $1,000-1,500$ vehicles in an hour.

Main criterion for determining service level of two-lane roads in Germany is average of speed of Sedan. Roads function is categorized into 6 level, from $A$ to $F$ [9]. in England, service level of two-lane roads is average speed, as same as Germany.

England ministry of transportation has determined different kinds of travel time-traffic volume functions for various links of roads in urban, suburban and intercity zones. We can use those functions for determining of average speed of network arch, analysis of capacity and service level [10].

In Iran, Mr. Alireza Adel, Msc candidate of university of Tehran, in the field of Road \& Transportation engineering; in his thesis has studied determination of capacity, after providing necessary information and statistics in two-lane roads of Guilan Province in 2002, and determined correction coefficients and statistics, and transformed them into information of ideal conditions. After that he drawn diagram of traffic-speed, acquired amount of capacity in ideal condition via confluence of lanes in stable running range, e.g. service level A,B, C, $\mathrm{D}$, and $\mathrm{E}$, and instable running, e.g. service level $\mathrm{F}$, which was less than suggested amount of HCM. He believed that this decreased is caused by lack of correct designing of road, lack of correct lining in middle and border of the road, driver's culture of people, ruinous asphalt surface in the road and wear and tear of vehicles.

Mr. Masoud Mohammadi, Msc candidate of Sharif university of Technology, in the field of Road \& Transportation engineering; in his thesis in title of "capacity analysis \& consideration of traffic function in special status of highway"; first of all considered different models of speeddensity, and after collecting necessary information \& statistics about Hemmat Highway; acquired the best model through analytical analysis of amount of speed, traffic and density by SPSS software.

Mr. Mohammad Tamannaei, student of Isfahan university of Technology, in Master's Degree, in the field of Road \& Transportation; in an essay in the title of "Analysis of basic capacity of highway" at first collected information about traffic volume, average speed and headway in Shahid Kharazi Highway of Isfahan in different times of day \& night; and calculated the capacity via microanalysis of traffic by headway and macro analysis of traffic volume by model of Greenburg \& Android. He found that this capacity is more than suggested amount of HCM. he stated that high percentage of divers elected non-safety headway and do not observe safety distance and cause to decrease average of distance headway and increasing of capacity.

The time and distance headway are microscopic parameters of traffic flow and are affected on the capacity, level of service and road safety [11]. In HCM2000manual, the time and distance headway and percent time spent following are considerable and effective parameters in capacity and service level, since in the previous methods of analysis of capacity in Iran, parameters of percent time spent following, time and distance headway not be investigated and also in the Iran's manual 161, not mentioned of these parameters, therefore in this study tried to further examined these parameters on capacity and service levels of two-lane road, and because the neural network methods have advantages over computing conventional methods such as no need to introduce specified function, capability of permanent correction, acceptance of noise at the input data, bearing of damages and failure, this method was chosen among other methods.

\section{A brief look at used fundamental in research}

1-3 for passing (other cars) in two-lane roads, those vehicles have low speed, using of pass-line in regions with sufficient view and proper distance between vehicles, is determined. If traffic limited area or geometrical design increase, passing (other cars) will decrease, so, we will have traffic [12]. 
Percentage of time, which vehicles lose because of traffic and lack of ability for passing (other cars) is called percent time spent following.

2-3- intellectual processing and high speed of human's brain in different fields caused that scientists try to find principles and fundamentals of human's brain and by modeling it, simulate an artificial nervous system, and transfer the ability of human's brain to computer.

Biologic nervous cell consist Dendrite, cell body and synapse. Dendrites are various branches of a nervous cell, cell body is main core of a biologic nervous cell, and data are processed there by cell body. Synapse is a place where two dendrites connect each other and in fact two different nervous cells connect to each other. At first dendrites receive information (signals) and transfer them to cell body, if signals are more than determined amount, cell body will be instigated and processes data and transfers new signals to output dendrite, thus, this dendrite will transfer the information to dendrite of adjacent cell.

Each nervous cell has formed from an input, output \& transfer function. Transfer function is responsible for signal processing, which has different kinds: tangent function, hyperbolic, logarithm...Each input multiply by an amount which named weight, the results are processed by transfer function. The Figs. 1 and 2 defines the structure of nervous cell and neural network algorithm. In some problems, by acting an amount on product, input in relevant weight, we can change algorithm (variable) of input to transfer function, which called net input. This amount cell Bias.

Kinds of nervous network is scalar and its amount is 1 , but advanced nervous network has vector form and includes $\mathrm{R}$ elements that each element multiply by its relevant weight. A network can have several layers; each layer has weight matrix (w), Bias matrix (b) and its special output (a). Different layer can have different neurons, but each layer has fixed amount of for bias vector. Output of each layer is input of next layer. The latest layer is named output layer and other layers are named hidden layers.

Nervous network does not need programming and is based on train. For training a pair of output $\&$ input is given to the system. One kind of algorithm is instruction of the Least Mean Square Error (LMS). LMS is performed based on decreasing of error between vectors of output and target. Amount of error is calculated by differences between these two parameters, e.g. vectors of output and target.

Following formula indicates average of LMS in LMS Algorithm:

$$
\begin{aligned}
& \mathrm{Q} \\
& \mathrm{mse}=(1 / \mathrm{Q}) \cdot \sum(\mathrm{t}(\mathrm{k})-\mathrm{a}(\mathrm{k}))^{2} \\
& k=1 \\
& \mathrm{a}(\mathrm{k}) \text { : output }
\end{aligned}
$$

This algorithm regulates weight and bias in such manner that LMS decreases to minimum amount. In this algorithm, at first a pair of target and input pattern is given to the network, inputs cause to create a signal in neurons of first layer, these signals create signal in neurons of next layer, it means that this process continue as a forward process from hidden layers to output layer, finally output of network comprise with target, and amount of errors will calculate. In this time accounting activities turns back and weights of output layer is modifies, and this action continue back ward up to weights of network input and first hidden layer modify. When amount of error decrease to acceptable amount and network weights has not significant changes, the network receives to stable status. Algorithm elects another pair of input-target vector and repeat above stages for it. Thus, other educational pairs (input-target) will enter and above stages repeat for all of them, this action named Epoch, it means that a series of calculating full operation for all educational pairs in this algorithm [3].

\section{Data collection}

Necessary statistics used available statistics in Road Maintenance Dept. in 2010. They elected statistics of different provinces in different conditions in order to have roads in smooth, hilly land and mountainous area, and also we have regions with different percentage of no passing. Roads of Fars, Boushehr, Khozestan, Kohkilouyeh, Kordestan, Zanjan, Qazvin, Qom, Mazandaran, Guilan and western Azerbaijan were elected. First of all a high traffic day in first 15 days of Farvardin (from 21st march to 4th April) \& a high traffic day in second 15 days of Shahrivar (from 22nd March to 6th September), which are top days for travel, were elected. After $3 \mathrm{~h}$, related top for morning, noon and evening in each day were determined. From primary statistics by using of Access software and indexes by-law 161, average speed, index of heavy vehicles, distributive index, time passing peak-distance and local passing peak-distance were determined. Characteristics of each road including average slope, lane width and shoulder
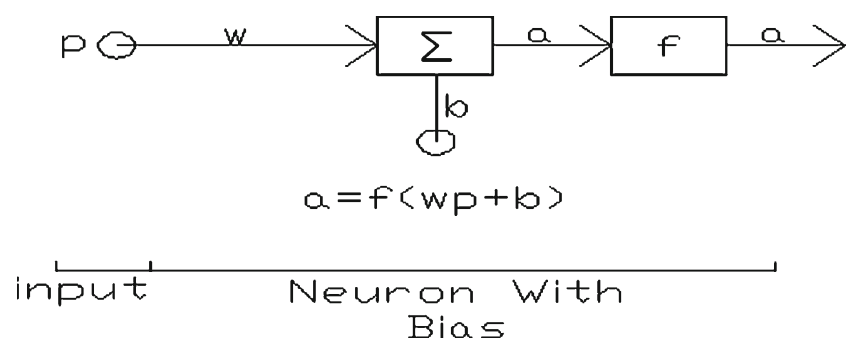

Fig. 1 Definition the structure of nervous cell [13] 


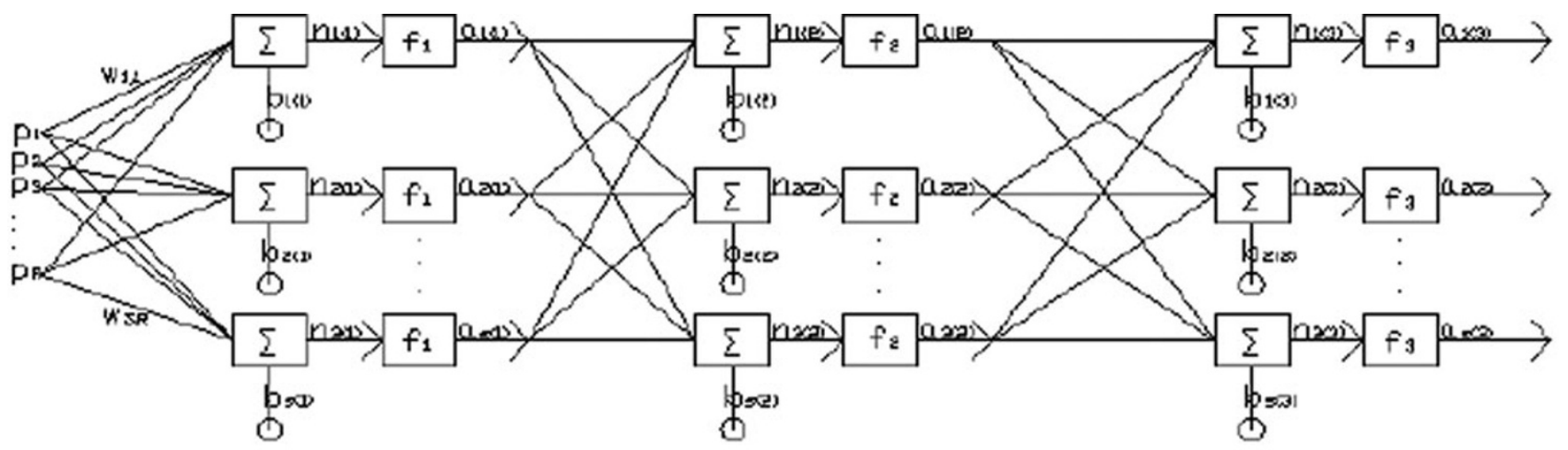

$a .1=f_{1}(w p+b)$

input Layer 1

$a_{\varepsilon}=f a(w p+b)$

Loyer 2 $a_{2}=f,(w p+b)$

Loyer 3

Fig. 2 The structure of neural network algorithm [13]

width were collected, their relevant indexes and also capacity of each road were determined. Percentage of following time average speed of travel was determined by HCM 2000.

Two-lane roads are categorized into 2 classes: I. Roads those users have favorite speed, like main roads and between cities roads. II. Road that speed is not important for users and use for local availability, like amusing and non-main roads. Service level in HCM for class I roads was determined based on Travel Average Speed ATS and percentage of following time PTSF, and for class II road based on percent time spent following (PTSF).

All elected roads in this research are class I.

\section{Used computer program}

MATLAB has many applications. One of its applications is in nervous network. Slope, index of heavy vehicle $\left(\mathrm{f}_{\mathrm{HV}}\right)$, distributive index $\left(f_{d}\right)$, index of correction of width of lane and shoulder $\left(f_{\mathrm{W}}\right)$, average speed $(\mathrm{v})$, percent time spent following.
Fig. 3 The result of best validation performance

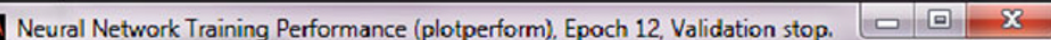

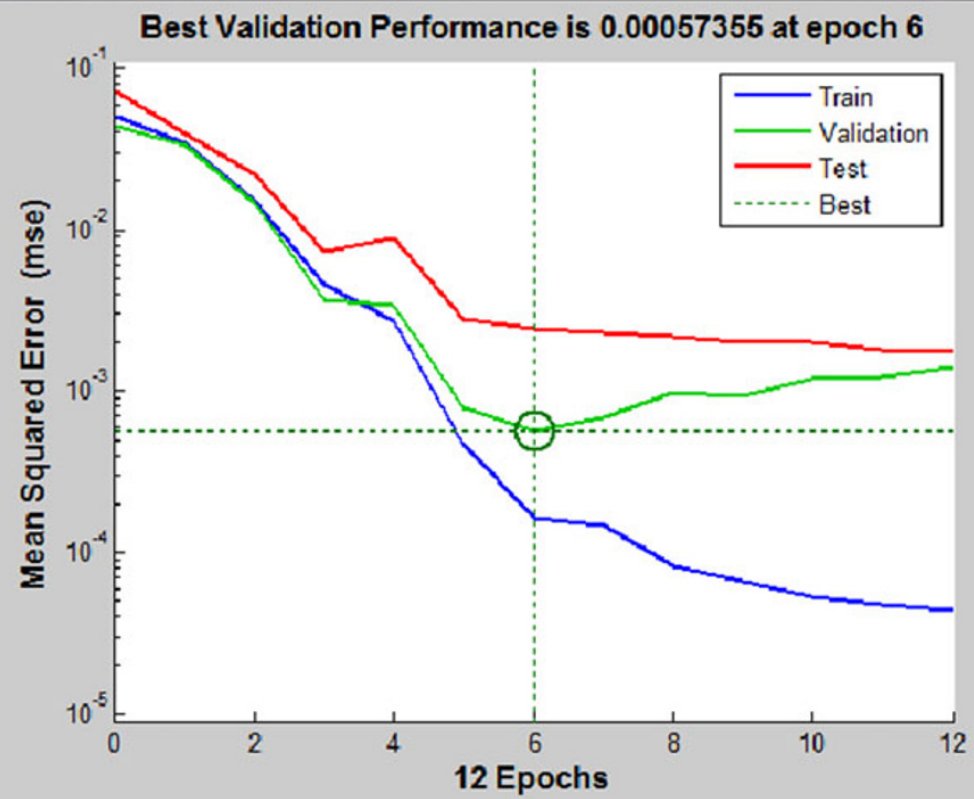


Fig. 4 Statistical indexes for validation test
Neural Network Training Regression (plotregression), Epoch 12, Validation stop.

Training: $\mathbf{R}=0.99221$
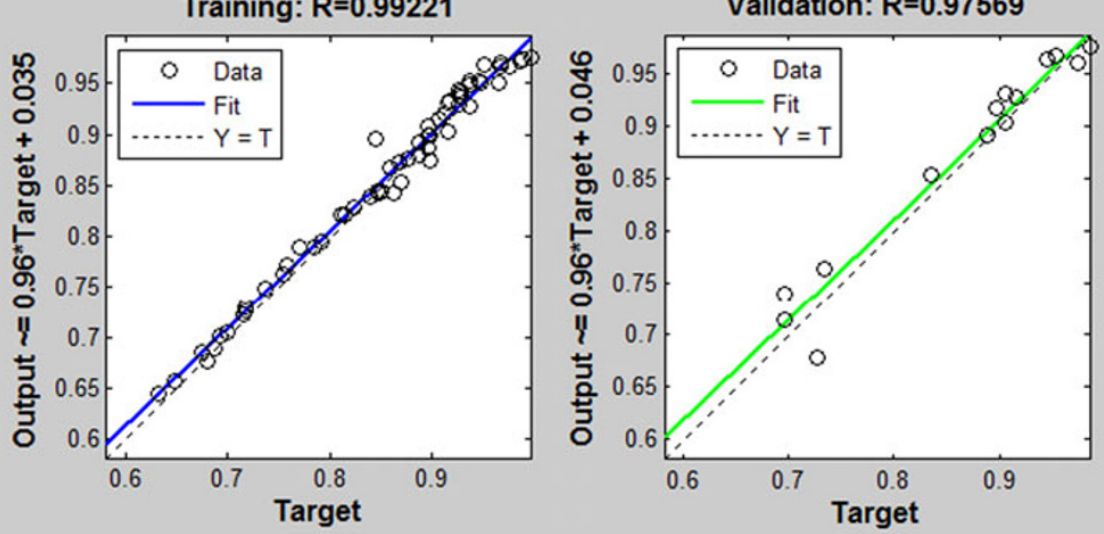

Test: $\mathbf{R}=0.97103$

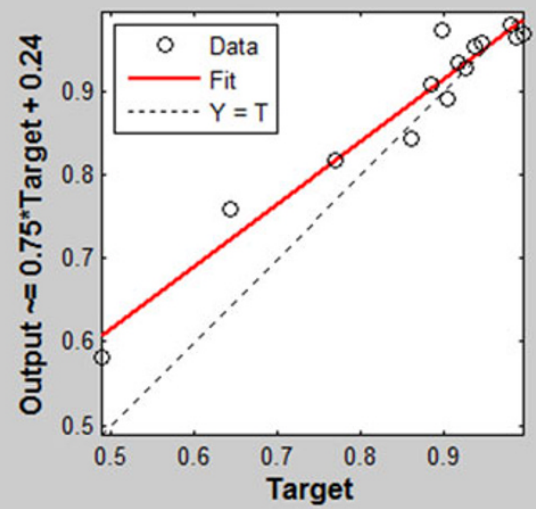

All: $\mathbf{R}=0.97753$

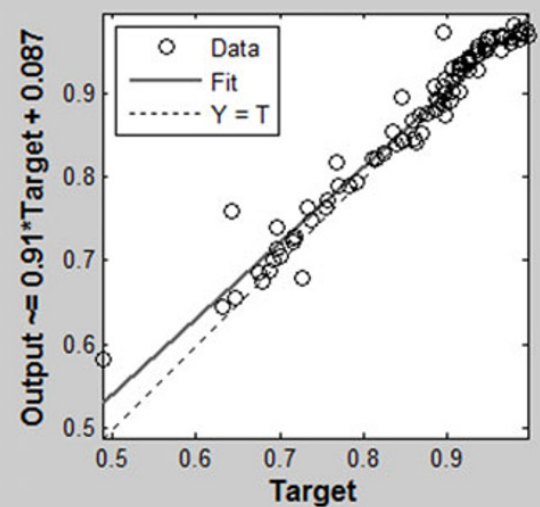

(PTSF), time passing peak-distance and local passing peak-distance that includes 8 cases, independent variable and capacity as dependent variable to nervous network are given.

\section{Results}

In nervous network by adding number of layers and different neurons, was designed various structures from nervous network. Nervous network selects some roads for network instruction, some for test and examine and some for network making credits. Ratio of the results by software to favorite results in each step is indicated by $\mathrm{R}$, if $\mathrm{R}$ be closer to $\mathbf{1}$ it means that obtained results by software is closer to observed result. Performance shows amount of error in education step. Whatever this error is less, it means that network education was better. Epoch has been completely described in clause 3. For different nervous networks, that network is favorite that receives to higher $\mathrm{R}$ and lower error by lower amount of epoch. Among them based on lower performance, the highest R (respectively, Rtrain, Rtest, Rvalid, Rall) and lowest epoch were selected.
The results of Figs. 3 and 4 show that the best network is structure of 3-layer 5-7-1 with following conditions:

$$
\begin{array}{r}
\text { performance }=4.33 \mathrm{e}^{\wedge}-5, \text { Rall }=0.97 \quad \text { Rvalid }=0.97 \\
\text { Rtest }=0.97 \quad \text { Rtrain }=0.99 \quad \text { epoch }=12
\end{array}
$$

Its related diagram is as follow:

The results of determining level of service based on ATS and PTSF based on HCM 2000, generally show that, service level based on ATS in comparison to service level based on PTSF is in better condition, it means that divers have higher

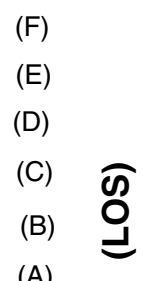

\section{(PTSF)}

Fig. 5 Consideration the effect of (PTSF) on level of service 
average speed and they almost pay no attention to traffic time spent following, so, safety is less and disasters are more. In Fig. 5, the effect of (PTSF) on level of service has been considered and the results show that decreasing PTSF cause to better LOS in stair form.

\section{Conclusion}

- Method of nervous network because of its abilities and characteristics like accept an error and noise in information, no need for introduction of function, no need to programming and complex planning, ability for modifying itself and tolerance of damages has predominance on any other methods.

- Among various structures of nervous network based on lowest performance, highest R (respectively, Rtrain, Rtest, Rvalid, Rall) and lowest epoch were selected.

The best network is structure of 3-layer 5-7-1 with following conditions:

$$
\begin{array}{r}
\text { performance }=4.33 \mathrm{e}^{\wedge}-5, \text { Rall }=0.97 \quad \text { Rvalid }=0.97 \\
\text { Rtest }=0.97 \quad \text { Rtrain }=0.99 \quad \text { epoch }=12
\end{array}
$$

- In determination of service level based on ATS and PTSF based on HCM 2000, generally, service level based on ATS in comparison to service level based on PTSF is in better condition, it means that divers have higher average speed and they almost pay no attention to traffic distance headway and time spent following, so, safety is less and disasters are more.
Open Access This article is distributed under the terms of the Creative Commons Attribution License which permits any use, distribution, and reproduction in any medium, provided the original author(s) and the source are credited.

\section{References}

1. Safari S (2000) "Analysis of traffic variables at highways and analysis on curve of speed-density", MA Thesis, Tehran University

2. Highway Capacity Manual (2000) Chapter20-two lane highway

3. Arabani M (2002) "Traffic engineering" Gilan University

4. Forschungsgesellschaft fur Stassen and Verkehrswesen (2002) "Handbuch fur die Beessung von Stassenverkehrsanlagen". Koln, Germany

5. Akcelik R (2008) The relationship between capacity and driver behavior. TRB National Roundabout Conference, Kansas City

6. Ross RP, Prassas ES, Mcshane WR (2004) "Traffic engineering", 3rd edn

7. Nicholas J, Laster A (2010) Traffic and highway engineering, 4th edn. Cengage Learning, University of Virginia

8. Capacity and Quality of Service of Two-Lane Highways, Transportation Research Board, National Research Council, NCHRP 3-55(3), November 1999

9. Wu N (1998) "The proposed new version of German Highway Capacity Manual". Proceeding of the International Conference on Traffic and Transportation Studies, Beijing, China, July 1998. ASCE, Reston, USA

10. A report from the Institute of Transportation Department of Transportation" instruction compilation of capacity of two-lane roads in Iran, Report of first stage

11. Arasan T (2003) "Headway distribution of heterogeneous traffic on urban arterials". J Inst Eng (India) 84, November 2003

12. Sadeghhosseini S (2002) "Time headway and platooning characteristics of vehicles on interstate highways", PhD. Thesis, University of Illinois, USA, 2002

13. Afshin S (1995) "Neural networks and its application in transportation". MA seminar of Transportation Planning, Elmosanat University winter 